\title{
STRUCTURAL RESPONSE OF MULTILAYERED ALUMINUM AND STEEL SPECIMENS SUBJECTED TO HIGH STRAIN RATE LOADING CONDITIONS
}

\author{
Danish Iqbal, Vikrant Tiwari \\ Indian Institute of Technology Delhi, Department of Applied Mechanics, New Delhi, India \\ e-mail:amz128262@iitd.ac.in; tiwariv@am.iitd.ac.in
}

\begin{abstract}
Series of experiments and a detailed computational analysis has been performed to investigate the high strain rate behaviour of homostacked Al 6063-T6 and IS 1570 alloys. Split Hopkinson pressure bar technique was utilized to study the effect of high rate loading on the stress strain relationship of single, double, tri and quad layered/stacked specimens. Three different specimen aspect ratios $1,0.75$ and 0.5 were also evaluated for different strain rates. A $2 \mathrm{~mm}$ thick pulse shaper was employed in achieving dynamic stress equilibrium, a near constant strain rate and a high rise time as per requirements. After analyzing the results from the experiments it was observed that single and halved specimens showed a close match in both the elastic and plastic regions for aluminium alloy as well as for steel. In the case of Al 6063-T6, a nearly bi-linear nature of the constitutive curve was observed for single and halved specimens, which transformed into near tri-linear nature for tri and quad stacked specimens. The dynamic numerical analysis showed a good agreement between the numerical and experimental results for a single and halved specimen in the case of $\mathrm{Al}$ alloy. For steel, a close correlation was observed for all the four cases.
\end{abstract}

Keywords: dynamic stress strain behaviour, multilayering, pulse shaper, finite element simulation

\section{Introduction}

Safety of a structure and its economics plays an important role in its designing process. Multilayer stacking and sandwiched structures are commonly used in structural applications where the required strength and material properties can be achieved at low financial cost. Such multi-layered structures can be commonly classified in two categories: First is homo-stacking where all constituent layers are made up of the same material of the same or different thickness. In the second category, constituent layers are made up of two or more different materials of the same or different thickness and can be called as hetro-stacked structures. Both these categories provides an important economical option of replacing a damaged layer instead of replacing the whole structure.

Some of the applications of multilayered stacking includes, but not limited to, thin walled compound cylinders, air-frames and layered armors. Such structures are commonly utilized in defence and space industries where they can be subjected to high rate loading applications, as in the case of vehicle crash or projectile impact/shock loading. Thus, it is warranted that dynamic mechanical response of such structures should be correctly evaluated and the effect of multi-layering is clearly understood. In the related research literature many specialized experimental setups are reported to test materials under such high rate loading conditions. One such an important technique is the Split Hopkinson Pressure Bar (SHPB) or the Kolsky bar which is also used in this work for the stated purpose. 
The split Hopkinson bar technique was initially developed by Kolsky (1949) to evaluate the material response at high strain rates. Since then SHPB and different numerical models have been utilized in dynamic characterization of a wide range of materials like metals, alloys, composites, ceramics etc. In one such an attempt, Jeng and Sheu (1994) investigated the dynamic response of Al 6061-O using SHPB and numerical modelling. They found that dynamic yield stress can be nearly $45 \%$ greater when compared with quasi-static conditions. In another study, Khan and Huang (1992) evaluated the dynamic response of Al 1100 alloy for a strain rate range of $10^{2}-10^{4} \mathrm{~s}^{-1}$. It was suggested that dislocation movements was the main reason behind the plastic flow of metals and was primarily responsible for the strain rate sensitivity.

With the advancements in technology, it is getting easier to conduct SHPB experiments. However, in most of the SHPB related studies researchers have expressed that maintaining the mandatory condition of force/stress equilibrium is a difficult task and needs to be verified. Wu and Gorham (1997) studied these requirements in detail and found factors that can adversely affect this equilibrium. Their results showed that inertia and wave propagation effects can cause a significant force difference between the two ends of the specimen.

In order to achieve force equilibrium and a near constant strain rate in the specimen, a technique called pulse shaping is commonly utilized. It requires a thin disc of metal to be placed at the impact end of the incident bar. Ellwood et al. (1982) modified the conventional SHPB setup by using a pre-loading bar and a dummy specimen as the pulse shaper. They suggested that the actual test specimen would be the best material to use as the pulse shaper. A substantially less variation in the strain rate was observed in the pulse shaped experiment.

Another important aspect that has been studied quite frequently is the geometrical effect or the effect of specimen dimensions on its dynamic response. In one such a study, graphite/epoxy composite specimens with varying $l / d$ ratios having square and rectangular cross sections were tested at high strain rates by Woldesenbet and Vinson (1999). They did not find any significant effect of either the aspect ratio or the specimen geometry on the stress-strain response of the material. However, in another similar study by Pankow et al. (2009) on the effect of specimen aspect ratio $(L / D$ ratio) and shape (cylindrical and square) on the stress-strain response of a material, it was observed that for some softer materials like aluminium, a smaller aspect ratio is advantageous. The study on the specimen cross section revealed that the stress-strain response did not differ much, although the plastic deformation in the case of cylindrical specimen was higher. Apart from the aspect ratio, interfacial friction between the specimen bar interfaces also influences the stress-strain response. Jankowiak et al. (2011) studied the effects of parameters like friction, inertia and elastic wave dispersion in many quasi-static and dynamic compression experiments. They used a friction correction and suggested that prior knowledge of the limiting value of friction was necessary for a correct numerical prediction. Li et al. (2009) observed that for $\mathrm{Al} 2024$ alloys the effect of specimen geometry and lubrication was in the same order of magnitude as strain rate sensitivity. The specimen aspect ratio can also trigger the formation of adiabatic shear bands in materials which show little or no effect of geometry on the stress strain response (Walley et al., 2006).

Highlighting the application of homolayered structures as protective barriers, many researchers (Corran et al., 1983; Radin and Goldsmith, 1988; Nia and Hoseini, 2011; Flores-Johnson et al., 2011) have conducted a series of investigations on different aluminium and steel alloys. They have evaluated the ballistic performance of a monolithic layer viz. different sequence of the homostacked configurations. These articles provide valuable insight into failure mechanisms and applicability of such structures. However, studies related to dynamic response characterization of such homostacked configurations are rare to find. In this article, an effort has been made to investigate the effect of high strain rate loading on homo-stacked specimens for two different materials, namely Al 6063-T6 and IS 5170 (mild steel). The specimens are evaluated at three different strain rates, and their true stress versus strain curves are analyzed and discussed. It 
should be noted that all experiments have been repeated at least three times for validating the repeatability of the specimen response. Numerical simulations using non-linear finite element software ABAQUS have also been carried out and their results are compared with the corresponding experiments.

\section{Experimental procedure}

\subsection{Split Hopkinson pressure bar}

The dynamic compressive experiments were performed on the split Hopkinson pressure bar apparatus based on the design provided by Engineers and Builders (2015). A schematic of the experimental setup is shown in Fig. 1.

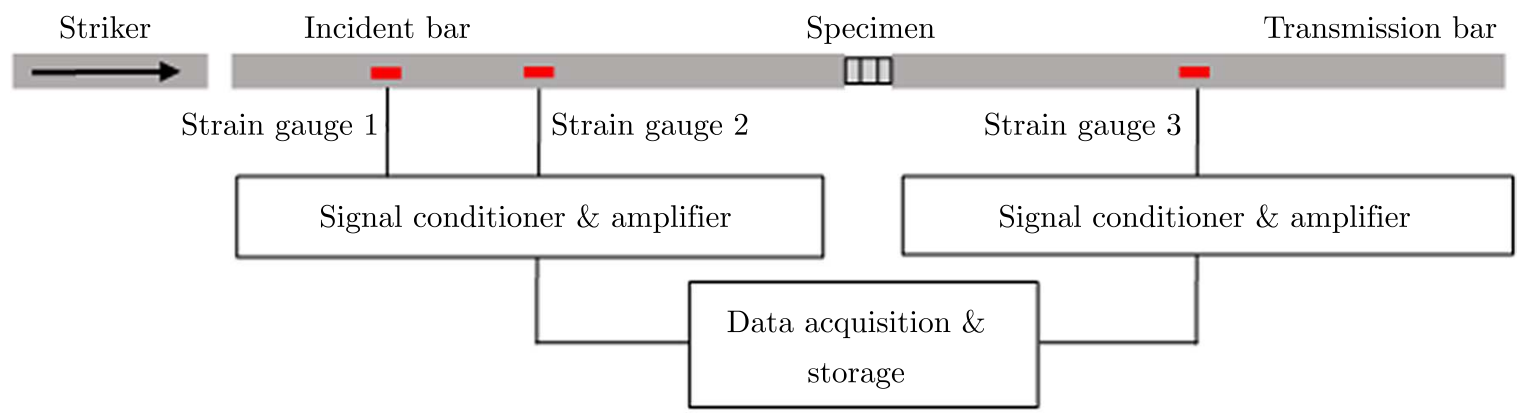

Fig. 1. Schematic of the split Hopkinson bar setup

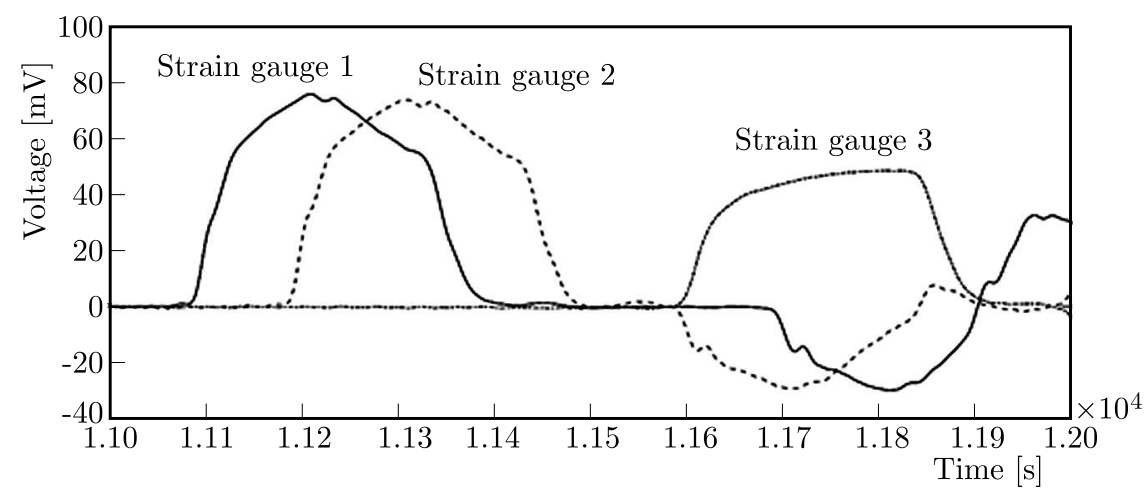

Fig. 2. A typical voltage signal obtained in SHPB experiment

The length of the striker bar and that of the incident and transmission bar was kept at $0.3 \mathrm{~m}$ and $2 \mathrm{~m}$ respectively. All bars were made of prismatic stainless steel rods of $20 \mathrm{~mm}$ diameter. Two diametrically opposite strain gauges $(120 \Omega)$ were mounted in a half bridge configuration at two positions (quarter and mid-length) on the incident bar and at the mid-length of the transmission bar. Two gauges were bonded on the incident bar to ascertain that there was no attenuation in the incident or reflected wave. Figure 2 shows typical voltage signals obtained in one of the experiments. Strain data from the gauges is later processed to determine the constitutive behaviour of the material. As the strain rate in the specimen is directly proportional to the reflected strain pulse $\varepsilon_{R}$, the average specimen strain rate is given as

$$
\dot{\varepsilon}=\frac{2 C_{b} \varepsilon_{R}}{h}
$$

where $C_{b}$ is the elastic wave speed in the bar and $h$ is the specimen length. Since the initial and final volume of the specimen remain constant, the average engineering stress $\sigma_{S}$ and strain $\varepsilon_{S}$ in the specimen is given by the following relation 


$$
\sigma_{S}(t)=\frac{A_{b}}{A_{s}} E_{b} \varepsilon_{T}(t) \quad \varepsilon_{S}(t)=2 \frac{C_{b}}{h} \int_{0}^{t} \varepsilon_{R}(t) d t
$$

where $A_{b}$ is the cross sectional area of the bars and $A_{s}$ is the cross sectional area of the specimen. $E_{b}$ is the elastic modulus of the bar material and $\varepsilon_{T}$ is the transmitted strain pulse.

\section{Specimen preparation and nomenclature}

Three sets of cylindrical disc specimens of varying aspect ratios of aluminium (Al 6063-T6) and mild steel (IS 1570) were fabricated from a single $11 \mathrm{~mm}$ diameter rods for both metals. This helped in avoiding slight property variations that might exist between different production batches. The chemical composition analysis of Al 6063-T6 and IS 1570 used in this study is given in Table 1.

Table 1. Chemical composition of Al 6063-T6 and IS 1570

\begin{tabular}{|l|c|c|c|c|c|c|c|c|c|}
\hline \multicolumn{10}{|c|}{$\mathrm{Al} 6063-\mathrm{T} 6$} \\
\hline Element & $\mathrm{Fe}$ & $\mathrm{Si}$ & $\mathrm{Cu}$ & $\mathrm{Mn}$ & $\mathrm{Al}$ & $\mathrm{Zn}$ & $\mathrm{Ni}$ & $\mathrm{Mg}$ & $\mathrm{Ti}$ \\
\hline \hline \% wt. & 0.3982 & 0.431 & 0.0069 & 0.014 & 98.55 & 0.0153 & 0.0058 & 0.5431 & 0.0056 \\
\hline \multicolumn{10}{|c|}{$\mathrm{IS} 1570$ (mild steel) } \\
\hline Element & $\mathrm{Fe}$ & $\mathrm{Si}$ & $\mathrm{Cu}$ & $\mathrm{Mn}$ & $\mathrm{Al}$ & $\mathrm{Cr}$ & $\mathrm{Mo}$ & $\mathrm{Ni}$ & $\mathrm{C}$ \\
\hline \hline \% wt. & 99.02 & 0.1349 & 0.1001 & 0.4073 & 0.0113 & 0.0556 & 0.0083 & 0.0613 & 0.111 \\
\hline
\end{tabular}

To avoid stress localization at the interfaces, it was ensured that specimen surface was flat and perpendicular to the sides. The machined specimens were first polished on a paper of grit designation 500, and then a progressively finer grit paper was used till a smooth surface was achieved with a grit paper of 800 . Both the contact surfaces of the specimen as well as the incident and reflected bars were lubricated for each experiment with a thin layer special Molybdenum Disulphide grease to reduce frictional effects. In case of layered specimens, the specimen-to-specimen interfaces along with the specimen-bar interfaces were also lubricated for each experiment. The specimen diameter utilized in this study was $11 \mathrm{~mm}$ and their thicknesses were $11,8.75$ and $5.5 \mathrm{~mm}$.

The stacking sequences for the specimens used in this study are shown in Table 2. The stacking scheme is divided into three specimen overall aspect ratios viz. 1, 0.75 and 0.5 which are further divided into smaller thicknesses of equal proportions as shown in Table 2. Specimens have been denoted with names according to their aspect ratio and the number of parts in which they are divided. For example, the specimen with 0.75 aspect ratio which is divided into 3 equal parts is named as 11-R-0.75-1/3, where 11 represents the specimen diameter, $R-x$ is the aspect ratio term and $1 / 3$ denotes the number of partitions.

Table 2. Specimen stacking sequence utilized in this study

\begin{tabular}{|c|c|c|c|c|}
\hline$L / D$ ratio & \multicolumn{4}{|c|}{ Specimen stacking scheme } \\
\hline \hline & $\square=L / D=1$ & $\prod \prod$ & $\prod \prod$ & $\prod \prod$ \\
\hline & $11 \mathrm{R}-1-1$ & $11 \mathrm{R}-1-1 / 2$ & $11 \mathrm{R}-1-1 / 3$ & $11 \mathrm{R}-1-1 / 4$ \\
\hline & $\square=L / D=0.75$ & $\prod$ & $\prod \prod$ & $\prod$ \\
& $\prod_{11 \mathrm{R}-0.75-1}$ & $11 \mathrm{R}-0.75-1 / 2$ & $11 \mathrm{R}-0.75-1 / 3$ & $11 \mathrm{R}-0.75-1 / 4$ \\
\hline
\end{tabular}




\subsection{Pulse shaping and dynamic force equilibrium}

When the pulse shaper deforms plastically on impact, it filters out the high frequency components of the incident pulse which are responsible for the dispersive nature of the incident wave. Different stages of the pulse shaper deformation for a typical case can be seen in Fig. 3 (Naghdabadi et al., 2012).

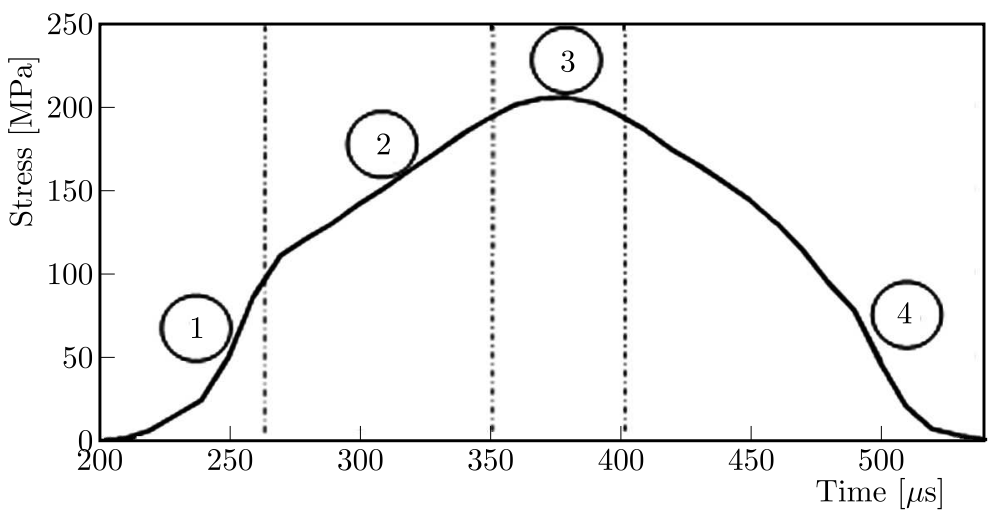

Fig. 3. A typical stress pulse obtained using a pulse shaper showing four stages of pulse shaper deformation

The initial stage of the process is dominated by elastic deformation of the pulse shaper on impact. Stage 2 represents plastic deformation of the pulse shaper. Stage 3 is the rigid mode and stage 4 is the elastic deformation during unloading of the pulse shaper (Naghdabadi et al., 2012). Early literature (Ellwood et al., 1982) shows that in order to obtain a constant strain rate deformation in the specimen it is beneficial to have an incident pulse with a nature similar to the anticipated stress-strain response of the specimen. This ensures that the stresses on both the faces of the specimen represent the average stress within the specimen which is an essential condition for any SHPB experiment. For the present study, a pulse shaper made of $2 \mathrm{~mm}$ thick discs of Al 6063 and IS 1570 were used. For each experiment in this study, it was verified that these conditions were satisfied so that governing equations (2.1) and (2.2) could be applied to determine the stress-strain history in the specimen. Experimental validation indicating that for all the four stacking sequences of the Al 6063 specimens force equilibrium is presented in Fig. 4.
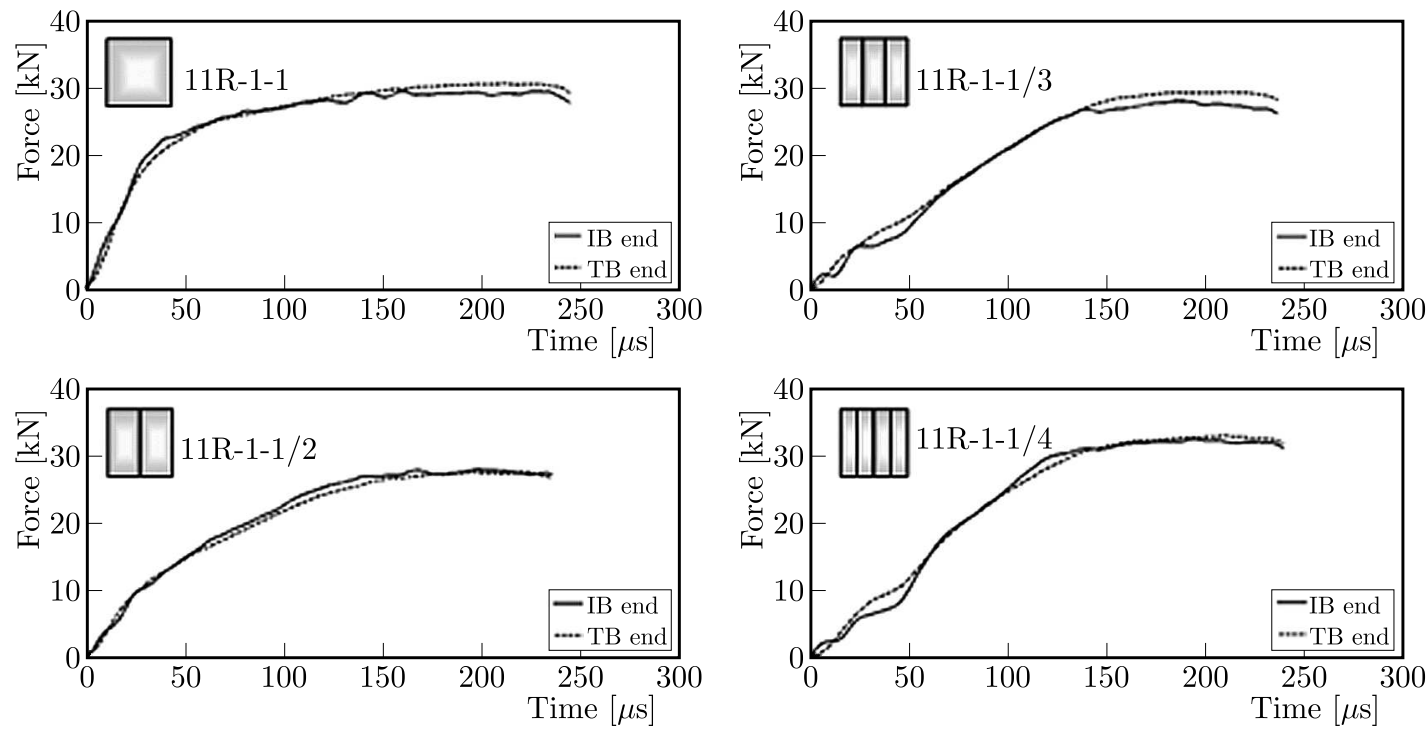

Fig. 4. Comparison of forces at the specimen interfaces, Incident Bar end (IB end) and Transmission Bar end (TB end) for all four stacking sequences 
The influence of the pulse shaper on the strain pulses and force ratio can be clearly seen in Fig. 5. It shows (Fig. 5a) voltage signals, variation of forces and force ratio (Fig. 5b) respectively for a typical experiment without the pulse shaper. Here, although the pulse is trapezoidal in shape, the forces on the two specimen bar interfaces do not match, which is also evident by the variation of the force ratio. In comparison, when a pulse shaper was used (Figs. 5c and 5d) it could be observed that there was a marked reduction in loading magnitude as compared to the experiment without the pulse shaper, but the loading time (pulse width) increased remarkably. This seems to ensure that the forces on the specimen faces are in dynamic equilibrium, as can been seen from Fig. 5d. Also, a significant increase in the rise time and the loading time of the pulse was observed, thus ensuring that the mandatory SHPB conditions of constant strain rate and dynamic stress equilibrium were satisfied. Similar results were also obtained for the IS 1570 .

(a)

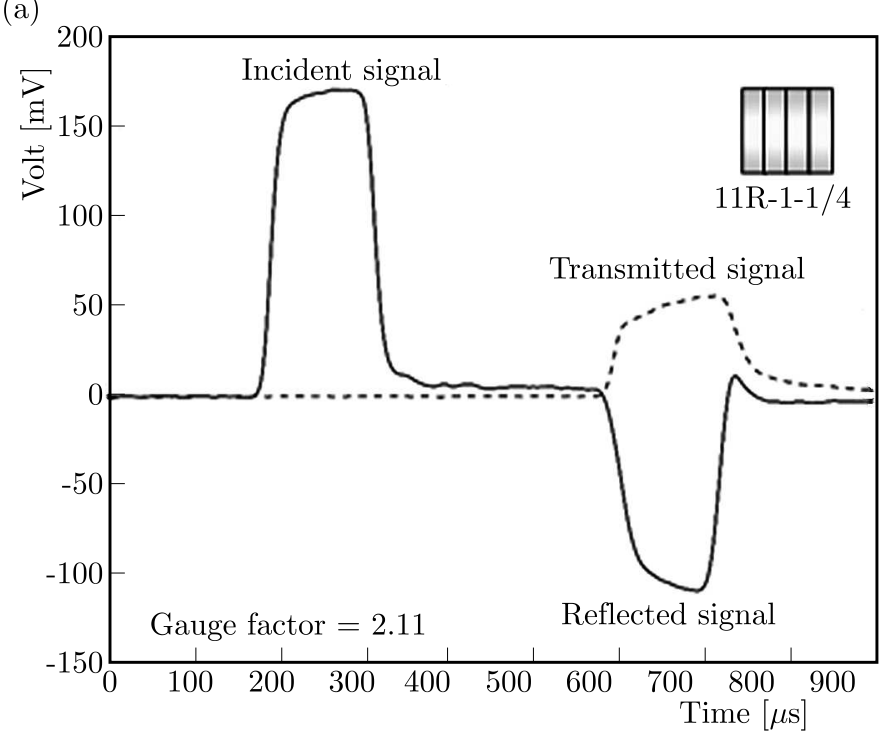

(c)

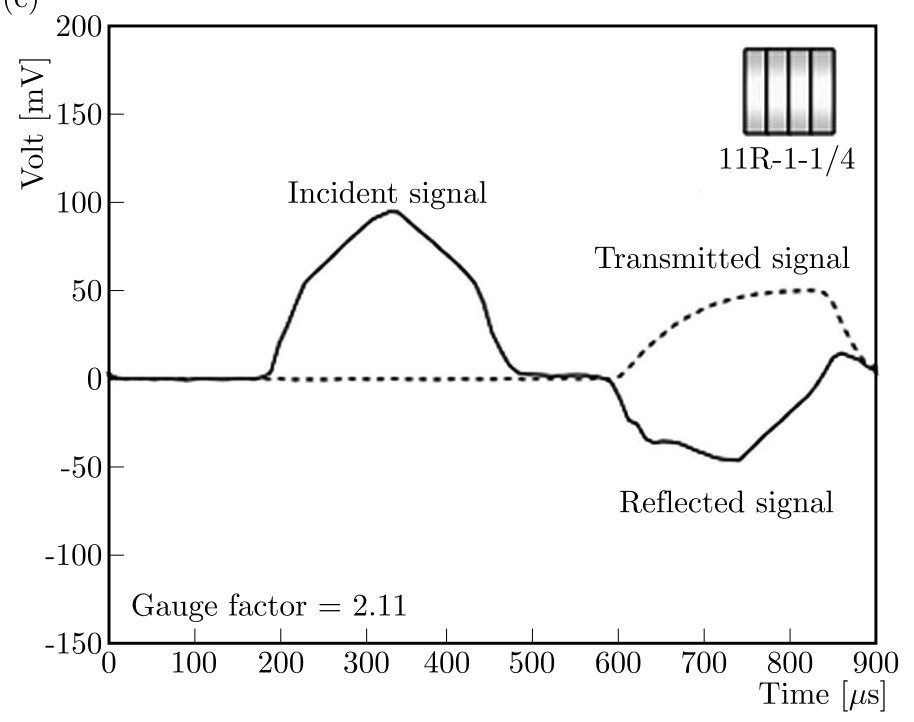

(b)

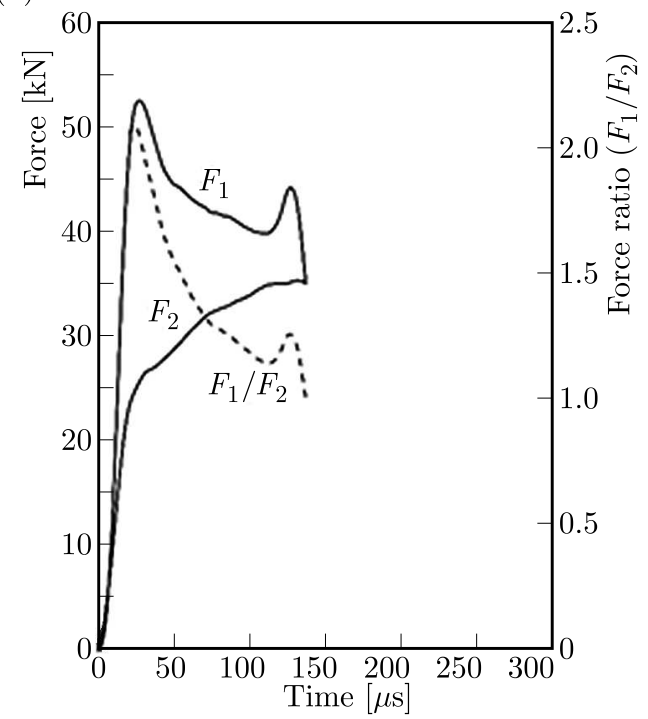

(d)

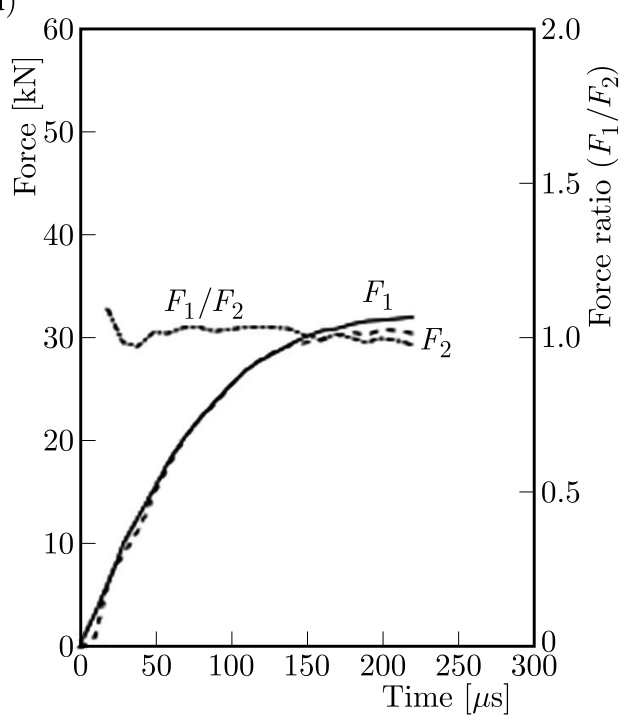

Fig. 5. (a) Voltage signals obtained without using a pulse shaper; (b) forces at specimen ends and the force ratio; (c) voltage signals obtained using the pulse shaper; (d) forces at specimen ends and the 


\section{Finite element simulations}

Non-linear 3D finite element simulations have been performed on ABAQUS/Explicit to numerically study the effect of specimen stacking on its stress-strain response in an SHPB experiment. The physical dimensions of the incident bar, transmission bar and specimen were kept same for all cases as those of the actual SHPB setup. The model had a bar diameter of $20 \mathrm{~mm}$ and a length of $2000 \mathrm{~mm}$. Since for a valid SHPB experiment the bars should remain elastic, a linear elastic model developed for isotropic elastic behaviour was used for the bars. The strain rate dependent behaviour of the specimen was modelled using the well-known Johnson-Cook model to evaluate its dynamic compressive response (Johnson and Cook, 1983)

$$
\sigma_{f l}=\left(A+B \varepsilon_{p l}^{n}\right)\left[1+C \ln \left(\frac{d \varepsilon_{p l} / d t}{\dot{\varepsilon}_{0}}\right)\right]\left[1-\left(\frac{T-T_{t}}{T_{m}-T_{t}}\right)^{m}\right]
$$

where $A, B, n, C$ and $m$ are material parameters. The J-C strength parameters used for aluminium and steel alloys are given in Table 3 . The parameters $A, B, n$ and $C$ were evaluated from a series of experiments performed. The value of thermal softening exponent $(m=0.89)$ was taken from the literature (Ye et al., 2016).

Table 3. Johnson-Cook parameters for Al 6063-T6 and IS 1570

\begin{tabular}{|l|c|c|c|c|}
\hline J-C Parameters & $A[\mathrm{MPa}]$ & $B[\mathrm{MPa}]$ & $n$ & $C$ \\
\hline \hline Al 6063-T6 & 156.3 & 284.55 & 0.12 & 0.0146 \\
\hline IS 1570 $(\mathrm{MS})$ & 225 & 352.65 & 0.4073 & 0.022 \\
\hline
\end{tabular}

To further improve the predictions of this numerical model, thermo-mechanical coupling effects were also incorporated. For this purpose, the increase in temperature $\Delta T$ was obtained from the following relation (Ye et al., 2016)

$$
\Delta T=\frac{\beta}{\rho C_{p}} \int \bar{\sigma} d \bar{\varepsilon}^{p l}
$$

here $\beta$ is the inelastic heat fraction, $\rho$ and $C_{p}$ are density and specific heat coefficients of the material. For simulations these parameters $C_{p}=851 \mathrm{~J} / \mathrm{kgK}$ and $\beta=0.9$ were incorporated from the literature (Sato et al., 1999).
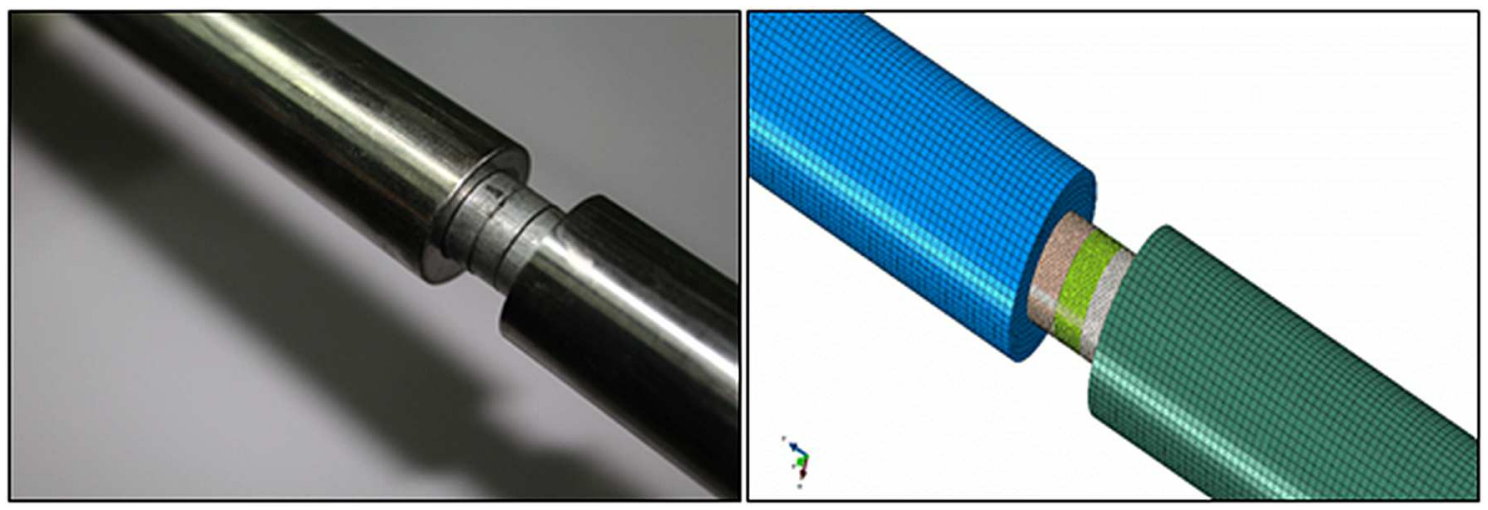

Fig. 6. Incident bar, specimen and transmission bar - experimental setup and FE model

The bars were meshed using 8 noded hexahedral brick elements (C3D8R), and the nodes along the axis of the bars were constrained radially. The total number of mesh elements generated on the incident and transmission bar were 29348 each, and the specimen with a much finer mesh (11R-1-1/3) had 23316 elements (Fig. 6). The interface between the specimen and bars was 
modelled as surface-to-surface contact. A penalty contact algorithm which allowed for treatment of more general types of contact and to enforce interfacial friction was utilized in the numerical simulations. A coefficient of friction of 0.05 was given to the interface between the bars and the specimen (Hartley et al., 2007). In the case of layered specimens, the specimen-to-specimen interface was given a coefficient of friction 0.03 (Hartley et al., 2007; Zhong et al., 2015).

The load was introduced in the form of the incident stress pulse at the free end of the incident bar. The strain data was measured on the nodes at the mid-points on the bar surfaces mimicking the actual experimental conditions. The SHPB equations were then employed to evaluate the true stress and strain data of the specimen under consideration.

\section{Results and discussion}

Dynamic compression tests were performed using the split Hopkinson pressure bar on stacked sequences of two selected materials viz. Al 6063-T6 and IS 1570 (mild steel) at different sets of strain rates. A single specimen of the aspect ratio 1 was sub-divided into 2, 3 and 4 equal parts, such that their individual aspect ratios $l / d$ were $0.5,0.33$ and 0.25 . On the same lines the specimens with the aspect ratio 0.75 and 0.5 were equally partitioned in 4 and 2 parts, respectively (Table 2). These specimens were then systematically evaluated under different sets of high strain loading conditions. Figures 7a-7c represent the true stress-true strain behaviour of $\mathrm{Al}$ 6063-T6 specimens with the aspect ratio 1, which are then further sub-divided into 2,3 and 4 layers of equal thickness, and evaluated under three different strain rates. The highest post yield stress is observed for the non-partitioned specimen at $500 \mathrm{~s}^{-1}$ and $1000 \mathrm{~s}^{-1}$ strain rates.
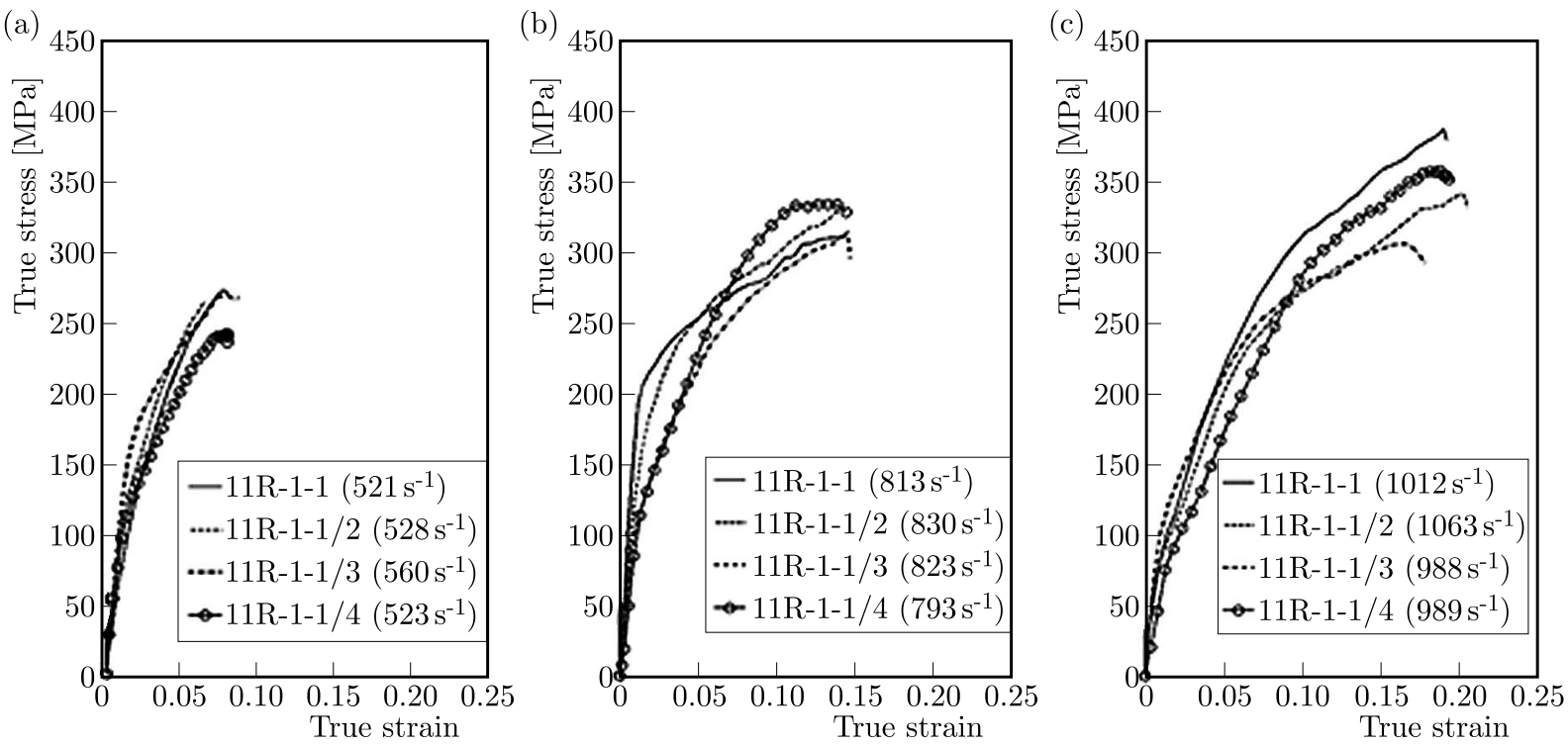

Fig. 7. (a), (b), (c) True stress versus true strain curves for different stacking sequences for Al 6063-T6 (11R-1-XX) at approximate strain rates of $500 \mathrm{~s}^{-1}, 800 \mathrm{~s}^{-1}$ and $1000 \mathrm{~s}^{-1}$

However, at the strain rate of $800 \mathrm{~s}^{-1}$, the specimen with 4 sub-divisions is observed to have the highest post yield stress. Figure 7 also reveals that there is a greater degree of similarity between the non-partitioned specimen and the specimen partitioned into half. Quantitatively, the peak stresses differ by $3 \%$ for $11 \mathrm{R}-1-1$ and $11 \mathrm{R}-1-1 / 2$ specimens and $9 \%$ for $11 \mathrm{R}-1-1 / 3$ and 11R-1-1/4 specimens. With a further increase in the number of sub-divisions, this similarity reduces. When the aspect ratio was reduced to 0.75 , it can be seen from Fig. 8 that the stress strain behaviour of the partitioned specimens was qualitatively closer to each other than that of the single specimen. This effect was found to be more pronounced at higher strain rates. 
However, at a lower aspect ratio of 0.5 , the plastic post yield stresses were both quantitatively and qualitatively closely matched, as can be seen in Fig. 9.
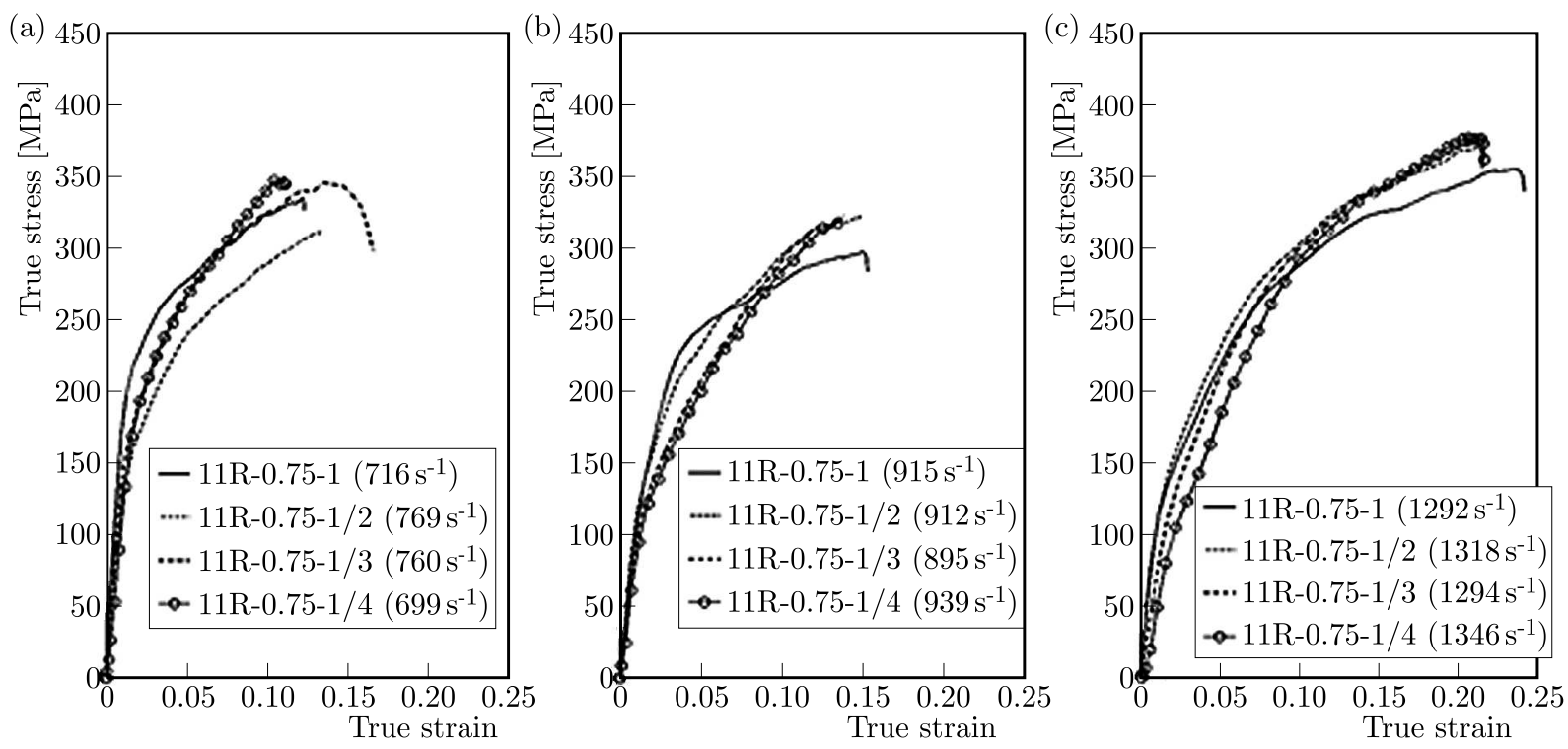

Fig. 8. True stress versus true strain curves for different stacking sequences for Al 6063-T6

(11R-0.75-XX) at approximate strain rates of (a) $700 \mathrm{~s}^{-1}$, (b) $900 \mathrm{~s}^{-1}$ and (c) $1300 \mathrm{~s}^{-1}$
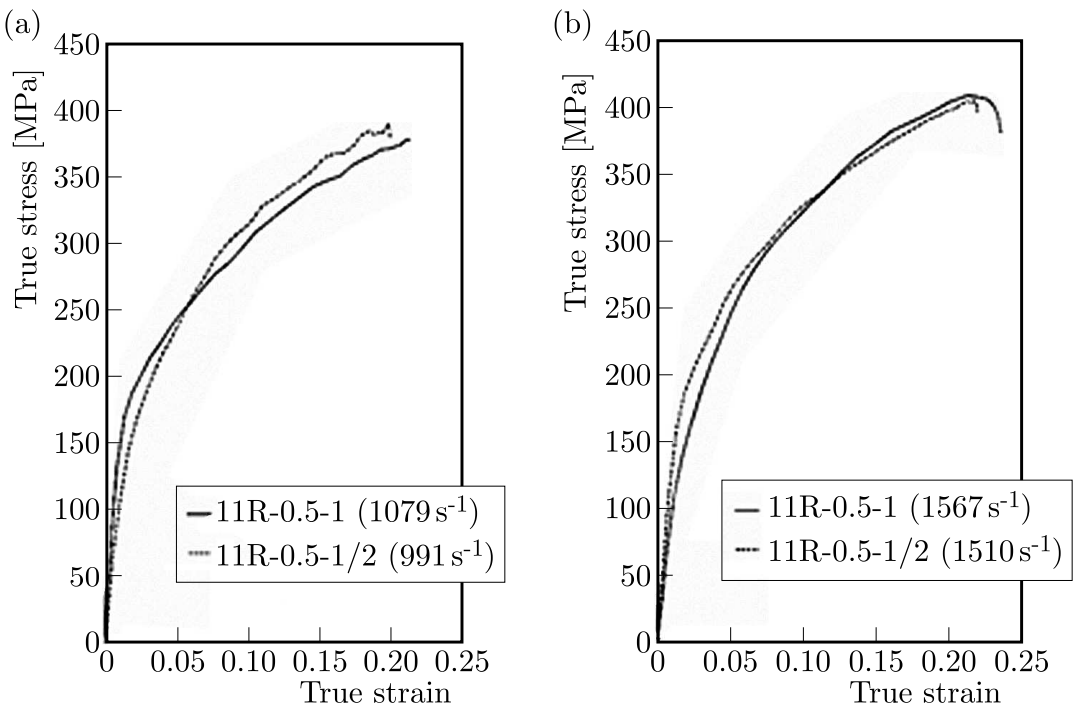

Fig. 9. True stress versus true strain curves for different stacking sequences for Al 6063-T6

(11R-0.5-XX) at approximate strain rates of (a) $1000 \mathrm{~s}^{-1}$ and (b) $1500 \mathrm{~s}^{-1}$

It can be observed that the difference in peak post yield stresses were $0.2 \%$ and $0.9 \%$ at $1000 \mathrm{~s}^{-1}$ and $1500 \mathrm{~s}^{-1}$, respectively. Experiments on the mild steel specimens were also performed at two different strain rates, $500 \mathrm{~s}^{-1}$ and $900 \mathrm{~s}^{-1}$. It was also observed that non-partitioned single specimens showed a characteristic upper and lower yield points as is generally seen in the quasi-static tests of mild steel (Fig. 10). However, this behaviour was not observed when the specimen was sub-divided in two, three and four layers. The highest post yield stress was observed for the specimen partitioned in two halves. The magnitudes of peak post yield stresses obtained were $662 \mathrm{MPa}$ and $725 \mathrm{MPa}$ at $500 \mathrm{~s}^{-1}$ and $900 \mathrm{~s}^{-1}$, respectively. The lowest peak post yield stress magnitudes were obtained for the specimen with three partitions as $602 \mathrm{MPa}$ and $687 \mathrm{MPa}$. If the slope of the individual curves in the plastic region are carefully observed in Fig. 10a, it is revealed that for non-partitioned and half partitioned specimens, the slopes match closely, both qualitatively as well as quantitatively. The maximum difference is about $2 \%$. Similarly, 
the difference in the magnitude of slope for one-third partitioned and one-fourth partitioned specimens is nearly $1.5 \%$. At higher strain rates, these magnitudes are found to be around $1.8 \%$ for single and halved specimens and $7 \%$ for one-third and one-fourth specimens, respectively. It is also observed that with an increase in the number of specimen partitions, the variation in the effective stiffness is more predominant in $\mathrm{Al} 6063$ in comparison to IS 1570.
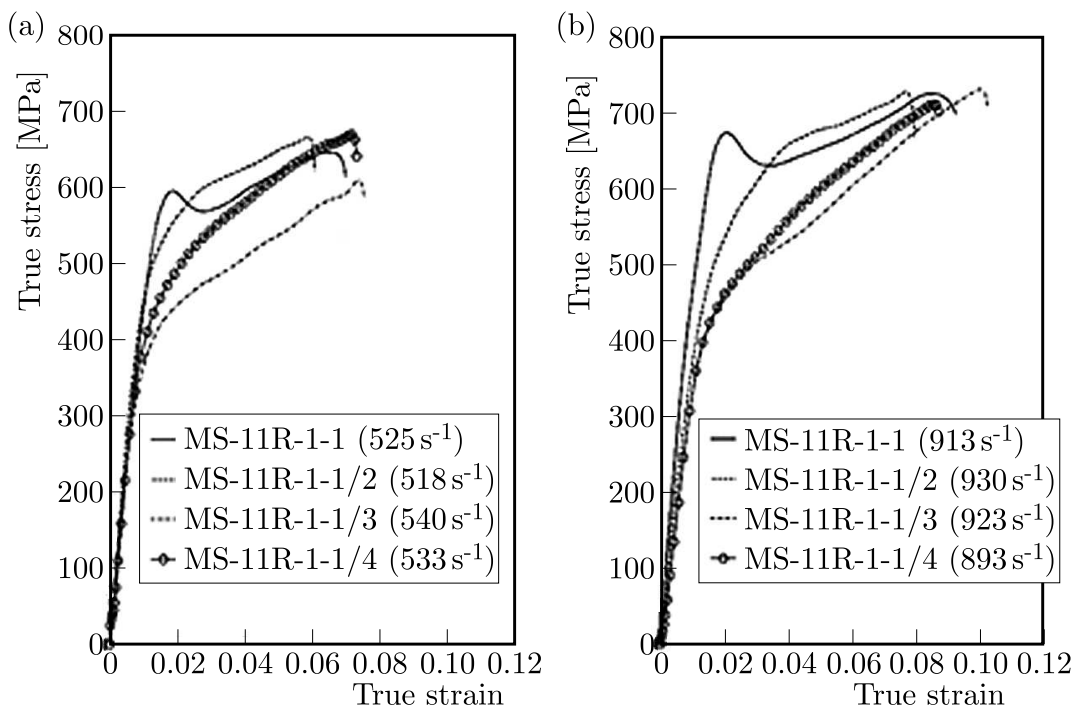

Fig. 10. True stress versus true strain curves for different stacking sequences for IS 1570 (mild steel)

(MS-11R-0.5-XX) at approximate strain rates of (a) $500 \mathrm{~s}^{-1}$ and (b) $900 \mathrm{~s}^{-1}$

Along with the experiments, finite element simulations were also performed for a different set of strain rates for $\mathrm{Al}$ 6063-T6 and IS 1570. Some of the results obtained are shown in Figs. 11 and 12 for $\mathrm{Al} 6063$ and IS 1570, respectively. For Al 6063 it, is observed that FE model incorporating the rate dependent Johnson-Cook model is able to correctly predict the true stress-strain response of the specimens for upto bi-partitions. However, when the same specimen is partitioned into tri and quad configurations, the $\mathrm{J}-\mathrm{C}$ model and the experiments exhibit poor correlation.

(a)

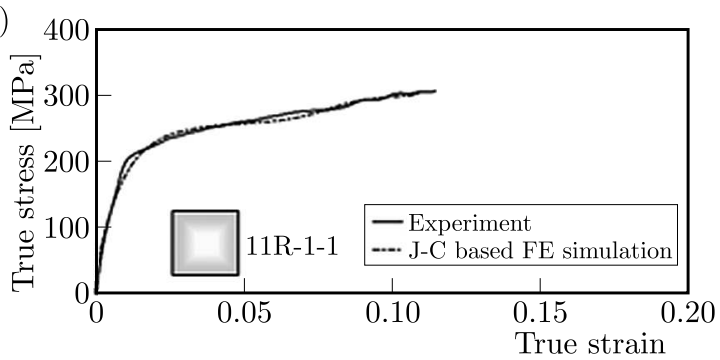

(c)

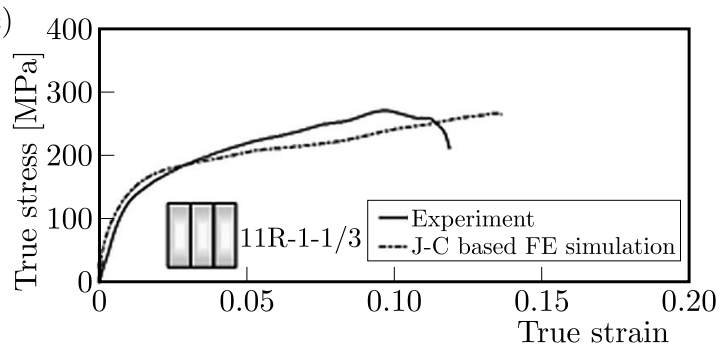

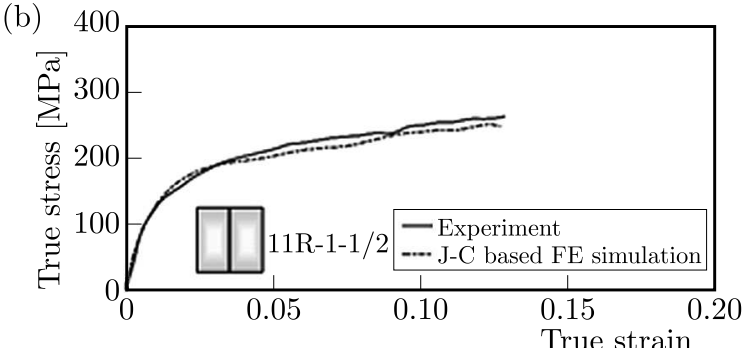

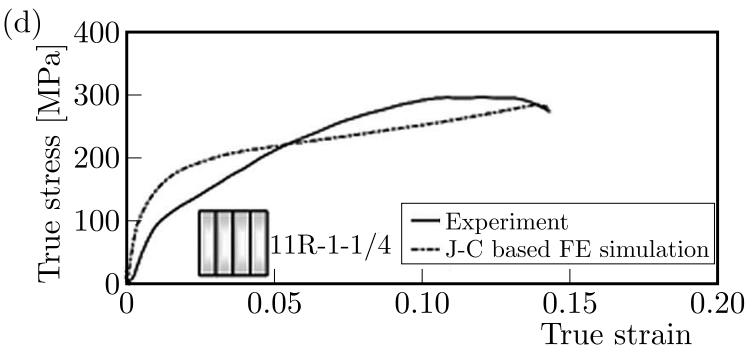

Fig. 11. (a)-(d) Comparison of experimental and FE simulation curves obtained for Al 6063-T6 $(11 \mathrm{R}-1-\mathrm{XX})$ at an approximate strain rate of $800 \mathrm{~s}^{-1}$ 
(a)

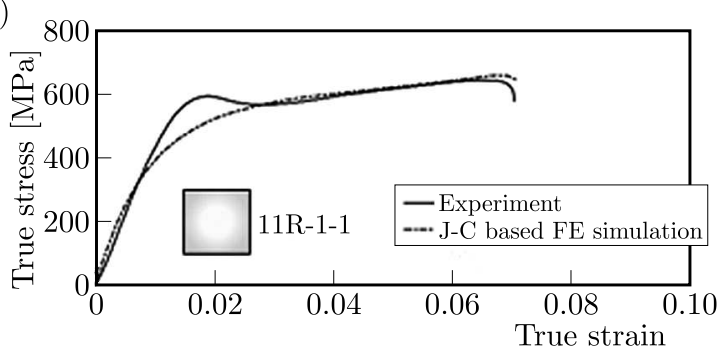

(c)

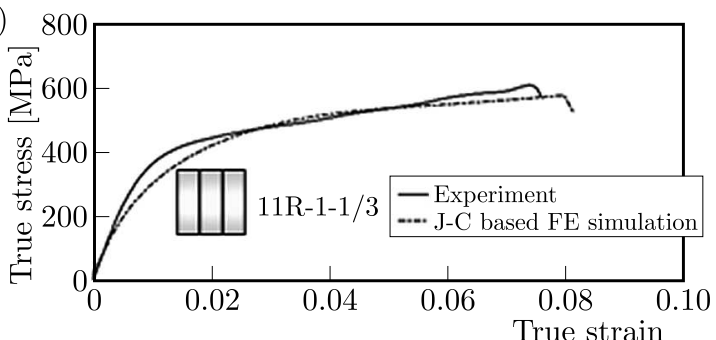

(b)

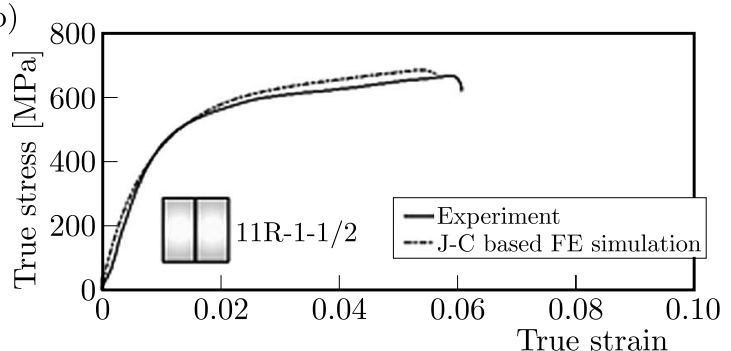

(d)

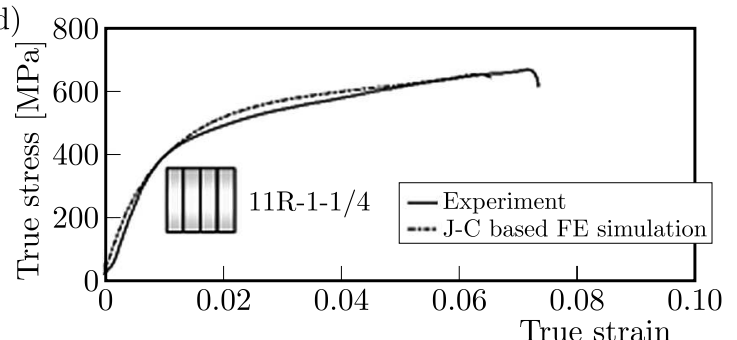

Fig. 12. (a)-(d) Comparison of experimental and FE simulation curves obtained for IS 1570

(MS-11R-1-XX) at an approximate strain rate of $500 \mathrm{~s}^{-1}$

In Figs. 11c and 11d, it can be clearly seen that the Johnson-Cook model is not able to correctly capture the response observed in the experiments for tri and quad partitioned specimens. In the case of mild steel, it can be observed from Fig. 12 that the J-C based FE model show a good agreement with the experimental results for all one, two, three and four partition configurations of the specimen. However, in Fig. 12a, it is also visible that J-C model could not completely capture the specimen response around the yield point region. It should be also noted that the authors observed no meaningful difference between the model predictions, with and without thermo-mechanical coupling. One such a result from FE simulation using thermomechanical coupling can be observed in Fig. 13 which shows the transient cross sectional view of the quad configuration (11R-1-1/4) of aluminium specimen at a strain rate of $500 \mathrm{~s}^{-1}$. Using FE simulations of Eq. (3.2), the maximum temperature gradient is found to be within $10 \mathrm{~K}$.

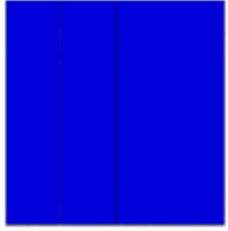

$t=0 \mathrm{~ms}$

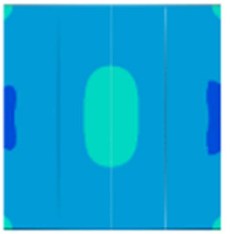

$t=0.100 \mathrm{~ms}$

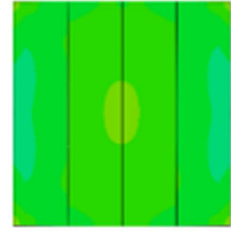

$t=0.150 \mathrm{~ms}$

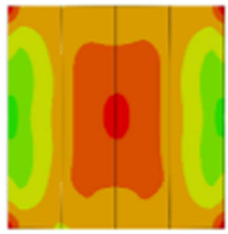

$t=0.225 \mathrm{~ms}$

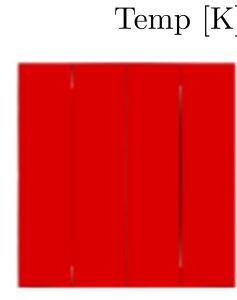

$t=0.275 \mathrm{~ms}$
$3.100 \mathrm{e}+2$

$3.050 \mathrm{e}+2$

$3.000 \mathrm{e}+2$

Fig. 13. Evolution of temperature in Al 6063-T6 specimen (11R-1-1/4) at a strain rate of $500 \mathrm{~s}^{-1}$

In the authors' opinion, even though the overall specimen response can be measured accurately, it is extremely difficult to predict the interlayer dynamic coefficient of friction for the stacked specimens. The difference between the experimental results and the numerical predictions can be attributed to so hard to quantify, complex and evolving boundary conditions at the interfaces. From the results, it is clearly evident that the mild steel specimens showed a comparatively higher strain rate sensitivity than the aluminium alloy at different strain rates. All the different layered mild steel specimens show a response that is relatively similar in nature to each other for the given strain rate. A single specimen when tested in dynamic compression showed an upper and lower yield point which is characteristic of mild steel when tested under the quasi-static condition. However, this behaviour was only limited to the non-partitioned specimens only. The finite element analysis, in the case of aluminium alloy, shows a good agreement 
with the experiments for specimens with zero and one partition. For the other two remaining cases, a clear difference in the slope, magnitude and the behaviour of the stress-strain curve is observed. In the case of FE simulations for IS 1570 mild steel specimens, a good correlation is achieved with the same material model for all the four cases. However, our simulations do not match the characteristic hump as can be seen in Fig. 13a.

The difference in the experimental and numerical predictions can be attributed to various factors like; (a) reliability of the value of dynamic coefficient of friction between the specimen-specimen and bar-specimen interfaces, (b) in experiments, even with the utmost care, it is impossible to create perfectly parallel surfaces of the specimen. While in the numerical model, perfectly parallel surfaces can be easily defined.

\section{Conclusions}

The present study involves experimental and numerical investigation of the behaviour of homolayered metallic media subjected to dynamic compressive loading. Two metallic alloys Al 6063-T6 and IS 1570 steel have been tested at different strain rates. The Al alloy under consideration exhibited a near tri-linear nature of the true stress-true strain curve when the specimen was partitioned into three and four parts. The above work can be concluded in the following points:

- This study has clearly shown that the dynamic response of homo layer stacking can be quite different than the monolithic layer specimen of the equivalent thickness.

- Design and selection of the pulse shaper is an important factor in evaluating the dynamic response of homostacked specimens. It is shown that by using a pulse shaper it is possible to apply the established technique like SHPB to characterize the stacked specimens.

- The current material model (Johnson-Cook) gives reasonably satisfactory match between numerical and experimental results for non-partitioned and bi-partitioned specimens. With an increase in the number of partitions, the similarity between the results decreases. Incorporating thermo-mechanical coupling does not yield any significant change in the model predictions. A better model capable of incorporating transient frictional boundary conditions and multiple wave reflections at the interfaces is required for evaluating further partitions.

\section{References}

1. Corran R.S.J., Shadbolt P.J., Ruiz C., 1983, Impact loading of platesan experimental investigation, International Journal of Impact Engineering, 1, 1, 3-22

2. Ellwood S., Griffiths L.J., Parry D.J., 1982, Materials testing at high constant strain rates, Journal of Physics E: Scientific Instruments, 15, 280-282

3. Engineers and Builders, 2015, Standard tools and techniques for dynamic characterization of materials, Design book, http://www.engineersandbuilders.com

4. Flores-Johnson E.A., Saleh M., Edwards L., 2011, Ballistic performance of multi-layered metallic plates impacted by a 7.62-mm APM2 projectile, International Journal of Impact Engineering, 38, 12, 1022-1032

5. Hartley R.S., Cloete T.J., Nurick G.N., 2007, An experimental assessment of friction effects in the split Hopkinson pressure bar using the ring compression test, International Journal of Impact Engineering, 34, 1705-1728

6. Jankowiak T., Rusinek A., Lodygowski T., 2011, Validation of the Klepaczko-Malinowski model for friction correction and recommendations on Split Hopkinson Pressure Bar, Finite Elements in Analysis and Design, 47, 10, 1191-1208 
7. Jenq S.T., Sheu S.L., 1994, An experimental and numerical analysis for high strain rate compressional behaviour of 6061-O aluminium alloy, Computers and Structures, 52, 1, 27-34

8. Johnson G.R., Cook W.H., 1983, A constitutive model and data for metal subjected to large strains, high strain rates and high temperatures, Proceedings of the Seventh Symposium on Ballistics, The Hague, Netherlands

9. Khan A.S., HuAng S., 1992, Experimental and theoretical study of mechanical behaviour of 1100 aluminium in the strain rate range $10^{-5}-10^{4} \mathrm{~s}^{-1}$, International Journal of Plasticity, 8, 4, 397-424

10. Kolsky H., 1949, An investigation of mechanical properties of materials at very high rates of loading, Proceedings of the Physical Society, B, 62, 11, 676-700

11. Li P., Siviour C.R., Petrinic N., 2009, The effect of strain rate, specimen geometry and lubrication on responses of aluminium AA2024 in uniaxial compression experiments, Experimental Mechanics, 49, 4, 587-593

12. Naghdabadi R., Ashrafi M.J., Arghavani J., 2012, Experimental and numerical investigation of pulse shaped split Hopkinson pressure bar test, Materials Science and Engineering A, 539, 285-293

13. Nia A.A., Hoseini G.R., 2011, Experimental study of perforation of multi-layered targets by hemispherical-nosed projectiles, Materials and Design, 32, 2, 1057-1065

14. Pankow M., Attard C., WaAs A.M., 2009, Specimen size and shape effect in split Hopkinson pressure bar testing, Journal of Strain Analysis for Engineering Design, 44, 8, 689-698

15. Radin J., Goldsmith W., 1988, Normal projectile penetration and perforation of layered targets, International Journal of Impact Engineering, 7, 2, 229-259

16. Sato Y.S., Kokawa H., Enomoto M., Jogan S., 1999, Microstructural evolution of 6063 aluminum during friction-stir welding, Metallurgical and Materials Transactions A, 30, 9, 2429-2437

17. Walley S.M., Radford D.D., Chapman D.J., 2006, The effect of aspect ratio on the compressive high rate deformation of three metallic alloys, Journal de Physique IV, 134, 851-856

18. Woldesenbet E., Vinson J.R., 1999, Specimen geometry effects on high strain rate testing of graphite/epoxy composites, AIAA Journal, 37, 9, 1102-1106

19. Wu X.J., Gorham D.A., 1997, Stress equilibrium in the split Hopkinson pressure bar test, Journal de Physique IV, 7, C3, 91-96

20. Ye T., Li L., Guo P., Xiao G., Chen Z., 2016, Effect of aging treatment on the microstructure and flow behavior of 6063 aluminum alloy compressed over a wide range of strain rate, International Journal of Impact Engineering, 90, 72-80

21. Zhong W.Z., Rusinek A., Jankowiak T., Abed F., Bernier R., Sutter G., 2015, Influence of interfacial friction and specimen configuration in Split Hopkinson Pressure Bar system, Tribology International, 90, 1-14 